\title{
Estudios ecológicos
}

\author{
Víctor Hugo Borja-Aburto, M.C., M.S.P., Ph.D.(I)
}

$\mathrm{U}$ no de los diseños de estudio más sencillos y frecuentemente empleados en la descripción de la situación de salud o en la investigación de nuevas exposiciones en poblaciones humanas son los estudios ecológicos. Sin embargo, por lo limitado de sus mediciones, pueden ser más susceptibles de sesgos que los estudios que se basan en observaciones individuales. En este trabajo revisaremos los conceptos generales de este tipo de diseños, las limitantes en la inferencia de sus resultados y presentaremos ejemplos de los mismos.

Los estudios ecológicos en epidemiología se distinguen de otros diseños en su unidad de observación, pues se caracterizan por estudiar grupos, más que individuos por separado. Frecuentemente se les denomina estudios exploratorios o generadores de hipótesis, dejando a los diseños experimentales y algunos diseños observacionales la característica de ser estudios etiológicos o probadores de hipótesis. Se les llama también diseños incompletos debido a que, por emplear promedios grupales, frecuentemente se desconoce la distribución conjunta de las características en estudio a nivel de cada individuo. ${ }^{1}$ Comúnmente las unidades de observación son diferentes áreas geográficas o diferentes periodos de tiempo en una misma área, a partir de las cuales se comparan las tasas de enfermedad y algunas otras características del grupo.

Es frecuente que ante las primeras sospechas de efectos negativos a la salud por algún producto o condición ambiental se exploren estas asociaciones en el ámbito grupal. Probablemente la principal motivación para los estudios ecológicos es la fácil disponibilidad de los datos; comúnmente se emplean datos registrados rutinariamente con propósitos administrativos o legales. Así, las instituciones gubernamentales tienen disponibles estadísticas de mortalidad y morbilidad, al igual que datos de los servicios de salud, mediciones ambientales, venta y consumo de productos de los cuales se sospecha algún efecto. El trabajo realizado por Yang y colaboradores ${ }^{2}$ para estudiar la relación entre cloración del agua y bajo peso al nacer es un ejemplo de lo anterior. Los autores compararon la frecuencia de bajo peso al nacer de hijos nacidos de mujeres primíparas en 14 municipios de Taiwán, donde $90 \%$ de la población era abastecida con agua clorada, con otros 14 municipios semejantes en sus características sociodemográficas y de urbanización, donde menos de $5 \%$ de la población era abastecida con agua clorada.

Otra motivación para los estudios ecológicos es que la comparación entre diversas áreas permite la evaluación de múltiples niveles de exposición, lo cual puede ser imposible en una sola área geográfica cuando se tienen exposiciones casi homogéneas. Siguiendo el ejemplo anterior, sería difícil hacer una comparación de niveles de exposición relevantes a los productos de la cloración entre las mujeres de un mismo municipio cuando la población comparte la misma fuente de suministro de agua.

Los estudios ecológicos han sido empleados por sociólogos y por epidemiólogos en diversas áreas, que van desde las enfermedades cardiovasculares hasta los efectos de la contaminación ambiental. Una revisión de los estudios ecológicos utilizados para investigar la disminución en el consumo de sal en la dieta y la reducción en la presión arterial, así como una

(I) Centro Nacional de Salud Ambiental, Instituto Nacional de Salud Publica, México.

Solicitud de sobretiros: Dr.Víctor Hugo Borja-Aburto, Centro Nacional de Salud Ambiental, Instituto Nacional de Salud Pública. Rancho Guadalupe s/n, 52140 Metepec, Estado de México, México. Correo electrónico: vborja@insp.mx 
comparación con los estudios a nivel individual y ensayos clínicos se puede consultar el trabajo de Law y colaboradores. $^{3}$

\section{Tipos de estudios ecológicos}

Frecuentemente, se clasifica a los estudios ecológicos como exploratorios, de grupos múltiples, de series de tiempo y mixtos. ${ }^{4,5}$

Estudios exploratorios. En los estudios exploratorios se comparan las tasas de enfermedad entre muchas regiones continuas durante un mismo periodo, o se compara la frecuencia de la enfermedad a través del tiempo en una misma región. En ninguno de los dos casos se hace una comparación formal con otras variables de los grupos, y el único propósito es buscar patrones espaciales o temporales que podrían sugerir hipótesis sobre las causas. Por ejemplo, Lee y colaboradores $^{6}$ estudiaron las tasas de melanoma maligno de piel y mostraron un incremento cercano a $3 \%$ por año en las poblaciones de Inglaterra, Canadá y los Estados Unidos de América (EUA), de 1951 a 1971. El análisis por cohorte de nacimiento mostró que este aumento se debe a un incremento en el riesgo de cáncer de piel en las cohortes de nacimiento más recientes. Este trabajo no propone hipótesis sobre los mecanismos de daño, pero sugiere la necesidad de realizar estudios epidemiológicos más detallados. En otro ejemplo, para mostrar la importancia del estudio de factores genéticos y ambientales, Croen y colaboradores ${ }^{7}$ estudiaron las variaciones en la prevalencia de labio leporino y paladar hendido de acuerdo con la raza de los padres y el lugar de nacimiento de la madre en el registro de nacimientos de California, EUA.

Estudios de grupos múltiples. Este es el tipo de estudio ecológico más común. En un estudio analítico de comparación de grupos múltiples se evalúa la asociación entre los niveles de exposición promedio y la frecuencia de la enfermedad entre varios grupos; comúnmente grupos geopolíticos. La fuente de datos suele ser las estadísticas de morbilidad y mortalidad rutinarias. Hatch y Susser ${ }^{8}$ compararon las tasas de cáncer de niños en relación con la exposición de radiación gamma en 69 pequeñas áreas geográficas cercanas a una planta nuclear. No se contó con información en el ámbito individual sobre la exposición a radiación, sólo se obtuvo información sobre las características sociodemográficas generales de las áreas en estudio.

Estudios de series de tiempo. En un estudio de series de tiempo analítico se comparan las variaciones temporales de los niveles de exposición (uso o consumo de productos o servicios, conductas, o concentraciones promedio de contaminantes, por ejemplo) con otra serie de tiempo que refleja los cambios en la frecuencia de la enfermedad en la población de un área geográfica. La inferencia causal de este tipo de análisis de series de tiempo puede ser limitada debido a cambios en los criterios diagnósticos de enfermedad y por dificultades provenientes de los periodos de latencia entre la exposición y los efectos o de la medición de la exposición. Loomis y colaboradores ${ }^{9}$ estudiaron la influencia de la contaminación del aire en la ciudad de México con los cambios diarios en un periodo de cuatro años. En este estudio documentaron una asociación entre las concentraciones de contaminantes y el riesgo de muerte prematura por enfermedades respiratorias.

Estudios mixtos. En esta categoría se incluyen los estudios de series de tiempo combinadas con la evaluación de grupos múltiples. Otra variante son los estudios que evalúan la exposición en el ámbito grupal y, en contraste, los datos de los efectos o enfermedad, así como posibles covariables, se encuentran disponibles en el ámbito individual. Hopenhayn-Rich y colaboradores ${ }^{10}$ compararon las tendencias de la mortalidad infantil en dos áreas geográficas de Chile: Antofagasta, con historia de contaminación natural por arsénico de las fuentes de abastecimiento de agua, y Valparaíso, una ciudad de exposición baja. La comparación de las variaciones en las fuentes de agua y la tendencia en la mortalidad infantil de las ciudades por medio de técnicas gráficas y modelos de regresión sugieren una influencia de la exposición a arsénico en el incremento del riesgo de muerte fetal y mortalidad infantil.

\section{Inferencia causal en los estudios ecológicos}

De manera clásica, en las escuelas de salud pública se enseña que no se debe confiar en los estudios ecológicos puesto que estos diseños son más susceptibles de presentar sesgos que los estudios basados en individuos y, más específicamente, están sujetos a la falacia ecológica. Este problema de hacer inferencias individuales a partir de estudios de grupo, clasificada por Selvin ${ }^{11}$ como "la falacia ecológica", ha ocasionado un problema de actitud en los epidemiólogos y un descrédito de estos estudios. Sin embargo, algunos practicantes de la salud pública defienden su utilidad real en las decisiones de prevención y control de enfermedades. Ejemplos clásicos, como los estudios de Snow y Farr sobre el cólera, ${ }^{12,13}$ muestran las bondades de estos estudios, a pesar de haber tenido limitantes en el entendimiento biológico detallado de la enfermedad. 
Entre otras cosas, este tipo de análisis recientemente ha mostrado, por ejemplo, el inicio de una posible epidemia de cáncer relacionada con la contaminación ambiental. ${ }^{14}$

Algunos autores como Susser ${ }^{15}$ sugieren que la justificación principal para el enfoque ecológico es estudiar la salud en un contexto ambiental, que la salud de un grupo es más que la suma de la salud de los miembros individuales, y que la perspectiva brindada por los estudios cuya unidad de análisis es el grupo más que los individuos, se debe entender como una manera de abordaje de la epidemiología y la salud pública, cuyo objeto de interés son los grupos. Este autor argumenta que la diferencia entre los diseños y enfoques descansa en los niveles de organización, ya que, en un nivel, las unidades individuales se integran en grupos y estos grupos se convierten en las unidades de análisis en el siguiente nivel. Cada nivel adquiere propiedades colectivas que son más que la suma de las propiedades de sus miembros individuales. El problema surge cuando se pretende extrapolar directamente de un nivel de análisis a otro, ya sea hacia arriba -de un gen a una molécula, a la célula, el tejido, el órgano, la persona, el grupo-, o en sentido inverso. ${ }^{15}$ Así, en el análisis de grupos las dimensiones de contexto o integrales son únicas y no pueden ser explicadas en el ámbito individual. Un ejemplo de lo anterior es la inmunidad de grupo en las enfermedades infecciosas, que se refiere a que la susceptibilidad, infección y transmisión de la infección no es independiente entre los individuos de un colectivo. Otro ejemplo es la modificación del efecto de variables individuales en el contexto de grupo, como pueden ser las conductas riesgosas para enfermedades de transmisión sexual en contextos con prevalencias altas o bajas de las enfermedades.

La falacia ecológica se refiere al error que se comete cuando se aceptan asociaciones entre eventos cuando en realidad no existen, siguiendo el supuesto de que los resultados obtenidos a partir de un estudio ecológico serían los mismos que se obtendrían de un estudio basado en observaciones de individuos (un supuesto implícito aquí es que el estándar de oro son los estudios de base individual). Este término en realidad cubre varias fuentes de sesgos ${ }^{16}$ que pueden afectar más a los estudios ecológicos que a los otros estudios con base individual. La mayor parte del sesgo ecológico se debe a la dificultad de controlar factores de confusión en este tipo de estudio. ${ }^{17} \mathrm{La}$ confusión, en este diseño, se refiere a variables que no son de interés intrínseco pero que se encuentran relacionadas con las medidas agregadas de la enfer- medad o exposición. La confusión en el ámbito individual y ecológico pueden ser independientes. Puede haber confusión en el ámbito individual entre los grupos por variables que no son confusoras a nivel ecológico, o viceversa. Por ejemplo, entre estados o municipios, puede ser que los ancianos tiendan a vivir en casas más contaminadas, lo que hace que se requiera controlar la edad como un confusor. Sin embargo, puede ser que el promedio de edad sea igual entre los distintos municipios, por lo que la edad no es un confusor en el ámbito ecológico. ${ }^{16}$

Adicionalmente, muchas veces los estudios ecológicos están sujetos a confusión potencial por la falta de medición de algunas covariables. Estas pueden ser covariables que rutinariamente no son registradas en los reportes oficiales. Por ejemplo, rutinariamente no se registra el número de fumadores en una comunidad, factor que podría ser relevante en el estudio de casi todas las enfermedades respiratorias.

Otro problema importante que se presenta en los estudios ecológicos es la determinación de la secuencia temporal entre la exposición y la enfermedad. La mayor parte de los estudios ecológicos son transversales, es decir, tanto la enfermedad como la exposición son medidas al mismo tiempo. Por ejemplo, si se desea evaluar la influencia del tabaco en una comunidad o de las medidas de prevención, se debe esperar un tiempo adecuado para observar sus efectos; es decir, tomar en cuenta el periodo de latencia entre la exposición y el efecto. Frecuentemente se asume que la exposición actual refleja la exposición en el pasado. El mismo problema ocurre en los estudios con base en individuos; sin embargo, en los estudios ecológicos se presenta la migración como problema adicional, es decir, la población afectada podría haber emigrado antes de la medición de la enfermedad, o algunos otros podrían haber inmigrado a la población estudiada, ocasionando un sesgo de selección. ${ }^{18}$

El problema de inadecuada especificación del modelo de análisis es otro factor de discrepancias cuando se espera que los resultados de los estudios ecológicos sean los mismos que de estudios individuales. Teóricamente, los resultados podrían ser los mismos cuando se emplean modelos lineales aditivos, sin términos multiplicativos. ${ }^{19}$ Sin embargo, en los estudios individuales la medida de asociación más frecuentemente empleada es una estimación de la razón de tasas o un equivalente como el riesgo relativo, mismo que no se puede estimar directamente en modelos de regresión con medidas de exposición continuas y ser interpretado como una medida de razón entre dos grupos de exposición. 


\section{Análisis estadístico de los estudios ecológicos}

La manera usual de evaluación de la asociación en estudios de grupos múltiples es mediante modelos lineales de regresión. Comúnmente se emplean los métodos de mínimos cuadrados para producir la ecuación de predicción: $Y=\beta_{0}+\beta_{1} X_{1}$, donde $\beta_{0}$ es el intercepto y $\beta_{1}$ la pendiente estimada o razón de cambio en la frecuencia de la enfermedad por unidad de cambio en la exposición. Las tasas o frecuencia de la enfermedad de cada región se convierten en la variable independiente, mientras que los promedios de las variables de exposición son las variables independientes. Cuando se cuenta con la medición de otras covariables (Z) éstas son incluidas en el modelo de regresión como una variable adicional para controlar por el posible efecto confusor de la misma, de tal manera que el modelo es $Y=\beta_{0}+\beta_{1} X_{1}+\beta_{2} Z_{2}$

Dependiendo del diseño y la distribución de los datos se pueden emplear otros modelos no lineales o no aditivos. Como las tasas de morbilidad y mortalidad en las regiones geográficas que se comparan comúnmente son eventos raros o que ocurren a bajas frecuencias, éstos semejan una distribución Poisson; así que la regresión Poisson puede ser usada para hacer las comparaciones entre los grupos y poder incluir variables potencialmente confusoras en modelos de regresión múltiple. El modelo se puede expresar de la siguiente manera $\log (E[Y])=\beta_{0}+\beta_{1} X_{1}+\beta_{2} Z_{2}$.

Un problema adicional es el poco desarrollo de modelos matemáticos que integren distintos niveles de análisis en estudios ecológicos donde se cuenta con variables integrales o contextuales medidas a nivel de grupo y con características en el ámbito individual. El análisis contextual, frecuentemente usado en ciencias sociales, es un ejemplo de estos modelos, y más recientemente los modelos de efectos mixtos permiten la evaluación de características combinadas en varios niveles, ya sea regiones, conglomerados e individuos. ${ }^{20}$

\section{El caso de la contaminación del aire y sus efectos en la mortalidad}

El caso de los estudios de los efectos de la contaminación del aire sobre la mortalidad puede ilustrar la aplicación de los diseños ecológicos. Uno de los primeros reportes que llamaron la atención sobre estos posible efectos se produjó en Londres, en 1952. Las condiciones meteorológicas provocaron una inversión térmica y una acumulación de contaminantes, principalmente de material particulado, que se asoció con un rápido aumento en el número de muertes en la ciudad, como se puede observar en la figura 1. Este patrón no dejó duda de que el incremento en la mortalidad se relacionó con la contaminación del aire en ausencia de epidemias por causas infecciosas. ${ }^{21}$ Por fortuna, las acciones de control de la calidad del aire han evitado que estos eventos de contaminación severa se presenten en épocas recientes. Sin embargo, durante la última década se han publicado un gran número de trabajos de estudios de series de tiempo en diversas ciudades que evalúan el efecto de la contaminación del aire a niveles mucho más bajos presentes en áreas urbanas de casi todo el mundo. ${ }^{22}$

En la ciudad de México se han realizado varios análisis de series de tiempo para evaluar este efecto. ${ }^{23-26}$ La figura 2 muestra el comportamiento de la mortalidad en la zona suroeste de la ciudad. Como se puede observar se presenta un componente estacional en el número de muertes, con picos en los meses fríos, de la misma manera que se comporta temporalmente la concentración de material particulado respirable. Sin embargo, la temperatura también tiene una variación similar, lo que podría indicar un efecto confusor importante, que ha llevado al desarrollo de modelos estadísticos que permiten evaluar el efecto de la calidad del aire, independientemente del efecto de la temperatura y otras condiciones ambientales que presentan variación estacional semejante. ${ }^{27}$

Este efecto de la contaminación del aire también ha sido evaluado con estudios ecológicos de comparación de grupos múltiples. Bobak y Leon ${ }^{28}$ condujeron un estudio ecológico sobre mortalidad infantil y contaminación del aire en la República Checa. Analizaron datos rutinarios de mortalidad infantil y contaminación del aire de 1986 a 1988 para 46 distritos de este país. Emplearon regresión logística para estimar los efectos de partículas suspendidas totales, dióxido

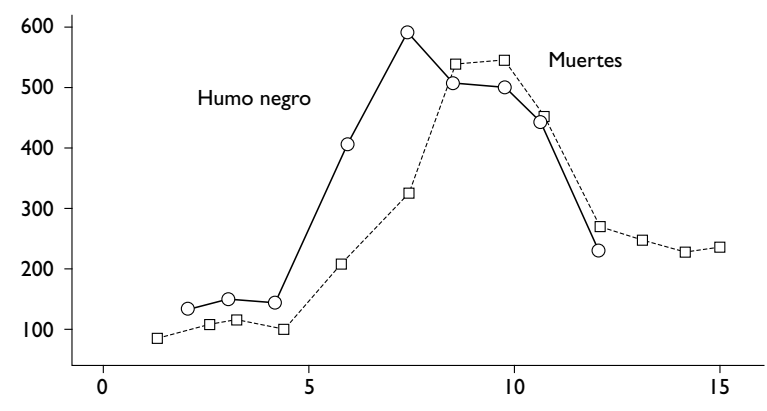

Figura I. Asociación entre el aumento de conTAMINACIÓN (MATERIAL PARTICULADO) E INCREMENTO DE NÚMERO DE MUERTES. LONDRES, $1952^{21}$ 

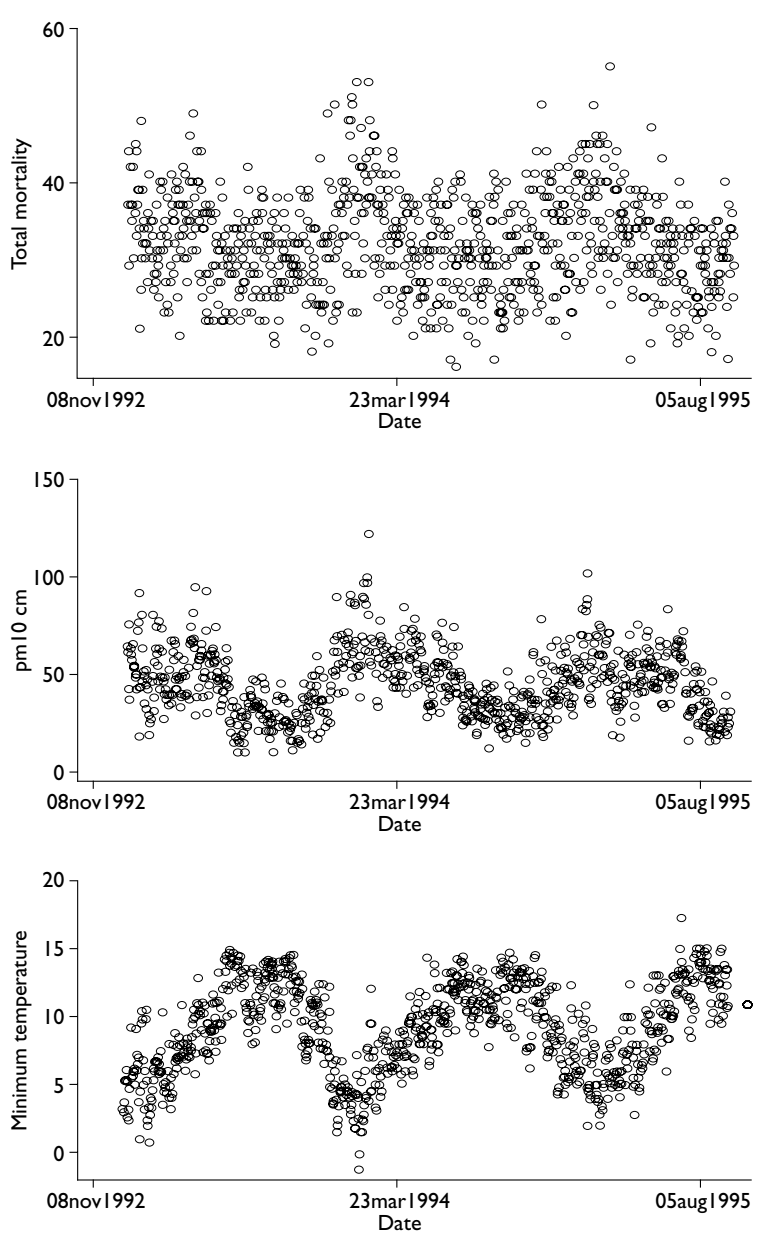

Figura 2. Comportamiento de la mortalidad en LA zona suroeste de LA CIUdAd de MÉxiCO $^{24}$

de azufre y óxidos de nitrógeno ajustando por características socioeconómicas de los distritos, tales como ingreso, posesión de automóviles y tasa de abortos. Los autores encontraron débiles asociaciones de partículas suspendidas menores a 10 micras con la mortalidad neonatal, pero una asociación más fuerte con la mortalidad posneonatal por causas respiratorias. Estos hallazgos fueron confirmados en un estudio de casos y controles más reciente. ${ }^{29}$ Loomis y colaboradores evaluaron también esta asociación en un estudio de series de tiempo en la ciudad de México, con resultados que apoyan las asociaciones iniciales del estudio de comparación de grupos múltiples. ${ }^{9}$

Uno de los problemas de estos estudios ecológicos es la medición de la exposición a los contaminantes. Por un lado, estas series de tiempo se basan en mediciones de la calidad del aire hechas con monito- res fijos, que no necesariamente reflejan las variaciones individuales en la exposición, derivadas del tipo de vivienda, tiempo dedicado a actividades extramuros o intensidad de las actividades y, por otro lado, la medición de las partículas suspendidas se basa en su peso más que en los componentes químicos de las mismas. Sin embargo, aunque estos estudios ecológicos no pueden por sí mismos confirmar la causalidad de los efectos observados, han impulsado una gran cantidad de otros estudios epidemiológicos y de experimentos en condiciones controladas con animales que cada día dan más pistas sobre los mecanismos que conducen a un aumento en el riesgo de morir en ambientes contaminados. A la fecha, tres estudios de cohorte han encontrado disminución en la esperanza de vida en ciudades con mayor concentración de partículas respirables. ${ }^{30-32}$

\section{Referencias}

I. Kleinbaum DG, Kupper LL, Morgenstern H. Epidemiologic research. Nueva York: Van Nostrand Reinhold, 1982.

2. Yang CY, Cheng BH, Tsai SS, Wu TN, Lin MC, Lin KC. Association between chlorination of drinking water and adverse pregnancy outcome in Taiwan. Environ Health Perspect 2000; 108:765-768.

3. Law MR, Frost CD, Wald NJ. By how much does dietary salt reduction lower blood pressure? I.Analysis of observatinal data among populations. BMJ I99|;302:8II-824.

4. Morgenstern H. Ecologic studies. En: Modern epidemiology. Rothman K, Greenland S, ed. Lippincott: Raven Publishers, 1998;459-480.

5. Morgenstern $\mathrm{H}$. Uses of ecologic analysis in epidemiologic research. Am J Public Health 1982;72(12): 1336-1344.

6. Lee JAH. Melanoma and exposure to sunlight. Epidemiol Rev 1982;4: 110-136.

7. Croen LA, Shaw GM, Wasserman CR, Tolarova MM. Racial and ethnic variations in the prevalence of orofacial clefts in California, 1983-1992. Am J Med Genet 1998;79(I):42-47.

8. Hatch M, Susser M. Background gamma radiation and childhood cancers within 10 miles of a U.S. nuclear plant. Int J Epidemiol 1990; 19: 546-552.

9. Loomis DP, Castillejos M, Gold DR, McDonnell W, Borja-Aburto VH. Air pollution and infant mortality in Mexico City. Epidemiology 1999; 10:118-123.

10. Hopenhayn-Rich C, Browning SR, Hertz-Picciotto I, Ferreccio C, Peralta $\mathrm{C}$, Gibb $\mathrm{H}$. Chronic arsenic exposure and risk of infant mortality in two areas of Chile. Environ Health Perspect 2000;108:667-673.

II. Selvin H. Durkheim's suicide and problems of empirical research. Am J Sociol 1958;63:607-619.

12. Snow J. On the mode of communication of cholera. 2a. edición. Londres: J. Churchill, 1855. Reprinted as Snow on cholera. Nueva York (NY): The common-wealth Fund, 1956.

13. Farr W. Report on the mortality of cholera in England in I848-1849. Londres: His Majesty's Stationery Office, 1852.

14. Poole C. Ecologic analysis as outlook and method. Am J Public Health 1994;84(5):715-716. 
15. Susser M. The logic in ecological: I. The logic of analysis. Am J Public Health 1994;84(5):825-829.

16. Steenland K, Deddens JA. Design and analysis of studies in environmental epidemiology. En: Topics in environmental epidemiology. Nueva York: Oxford University Press, 1997.

17. Greenland S, Robins J. Ecologic studies-biases, misconceptions, and conterexamples. Am J Epidemiol 1994;139(8):747-760.

18. Polissar L. The effect of migration on comparison of disease rates in geographic studies in the United States. Am J Epidemiol 1980; I I (2): 175-182.

19. Piantadosi S. Ecologic biases. Am J Epidemiol 1994;139(8):761-764.

20. Goldstein H. Multilevel statistical models. 2a. edición. Londres: Edward Arnold, 1995.

21 . Schwartz J.Air pollution and daily mortality:A review and meta-analysis. Environ Res 1994;64:36-52.

22. Levy JI, Hammitt JK, Spengler JD. Estimating the mortality impacts of particulate matter:What can be learned from between-study variability? Environ Health Perspect 108:109-II7(200a).

23. Borja-Aburto VH, Loomis D, Shy C, Bangdiwala S, Rascón-Pacheco RA. Ozone, suspended particulates and daily mortality in Mexico City. Am J Epidemiol 1997;145:258-268.

24. Borja-Aburto VH, Castillejos M, Gold DR, Bierzwinski S, Loomis D. Mortality and ambient fine particles in Southwest Mexico City, 1993- 1995. Environ Health Perspect 1998; 106:849-855.
25. Castillejos M, Borja-Aburto VH, Dockery DW, Gold DR, Loomis D. Airborne coarse particles and mortality. Inhal Toxicol 2000; I2 (Suppl I): $61-72$.

26. Téllez-Rojo MM, Romieu I,Velasco S. Daily respiratory mortality and pollution in Mexico City. The importance of considering place of death. Eur Respir J 2000;16(3):391-396.

27. Samet JM, Zeger SL, Berhane K. Particulate air pollution and daily mortality. Health Effects Institute, August 1995.

28. Bobak M, Leon DA. Air pollution and infant mortality in the Czech Republic, 1986-88. Lancet 1992;340:1010-1014.

29. Bobak M. Leon DA. The effect of air pollution on infant mortality appears specific for respiratory causes in the postneonatal period. Epidemiology 1999;10(6):666-670.

30. Abbey DE, Nishino N, McDonnell WF, Burchette RJ, Knutsen SF, Beeson WL et al. Long-term inhalable particles and other air pollutants related to mortality in nonsmokers. Am J Respir Crit Care Med 1999; 159: 373-382.

3I. Dockery DW, Pope CA III, Xu X, Spengler JD, Ware JH, Fray ME et al. An association between air pollution and mortality in six US cities. $N$ Engl J Med 1993;329:1753-1759.

32. Pope CA III,Thun MJ, Namboodiri MM, Dockery DW, Evans JS, Speizer FE et al. Particulate air pollution as a predictor of mortality in a prospective study of US adults. Am J Respir Crit Care Med 1995;141:669-674. 